\title{
Differential Anticancer Activity of Pterostilbene Against Three Subtypes of Human Breast Cancer Cells
}

\author{
REI WAKIMOTO, MISAKI ONO, MIKAKO TAKESHIMA, TAKAKO HIGUCHI and SHUJI NAKANO \\ Graduate School of Health and Nutritional Sciences, Nakamura Gakuen University, Fukuoka, Japan
}

\begin{abstract}
Although pterostilbene, a natural analog of resveratrol, has potent antitumor activity against several human cancer types, the possible inhibitory mechanisms against subtypes of human breast cancer with different hormone receptor and human epidermal growth factor receptor 2 (HER2) status remain unknown. We investigated the anticancer activity of pterostilbene using three subtypes of breast cancer cell lines. Pterostilbene treatment exhibited a dose-dependent antiproliferative activity, with the greatest growth inhibition observed in triple-negative MDA-MB-468 cells. Although pterostilbene arrested cell-cycle progression at the $G_{0} / G_{1}$ phase regardless of breast cancer subtype, its apoptosis-inducing activity was highly apparent in MDA-MB468 cells. Pterostilbene induced strong and sustained activation of extracellular signal-regulated kinase (ERK) $1 / 2$, with concomitant cyclin D1 suppression and 21 up-regulation, and inhibited the phosphorylation of AKT and mammalian target of rapamycin (mTOR), followed by subsequent upregulation of $B A X$ without affecting $B$-cell lymphoma-extra large $(B C L-x L)$. Oral administration of pterostilbene significantly suppressed tumor growth in nude mice xenotransplanted with MDA-MB-468 cells. These data suggest a potential role of pterostilbene for prevention and treatment of human breast cancer, especially of triple-negative breast cancer.
\end{abstract}

Breast cancer is the most frequently occurring cancer among women, and the leading cause of cancer death worldwide (1). Breast cancer is a heterogeneous disease that can be classified into several distinct subtypes based on estrogen receptor (ER), progesterone receptor (PR), and human epidermal growth

Correspondence to: Shuji Nakano, Graduate School of Health and Nutritional Sciences, Nakamura Gakuen University, 5-7-1 Befu, Johnan-ku, Fukuoka 814-0198, Japan. E-mail: snakano@nakamurau.ac.jp

Key Words: Breast cancer, pterostilbene, cell cycle, $\mathrm{G}_{0} / \mathrm{G}_{1}$ arrest, apoptosis. factor receptor 2 (HER2) expression. Therapeutic options for patients with advanced breast cancer are limited and depend on the breast cancer subtype. Although survival of patients with breast cancer has been improved by hormonal and targeted therapy in recent years, triple-negative breast cancer, which is clinically negative for expression of ER, PR and HER2, is associated with a poor prognosis (2). Because there are a limited number of treatments for triple-negative breast cancer, development of novel treatment options and prevention for this type of breast cancer is extremely important. In this context, fruit and vegetables have attracted attention because their intake has been shown to reduce the risk of breast cancer (3), and differentially affect ER-negative and -positive breast cancer incidence rates, preferentially lowering the risk of ERnegative breast cancer in postmenopausal women (4). Furthermore, a recent cohort study showed that fruit intake in adolescence significantly reduces the risk of ER-negative, but not ER-positive premenopausal breast cancer (5). Such differential cancer-preventive potentials of fruit and vegetables are considered to be attributable to phytochemicals, since a growing number of studies have uncovered potential applications for phytochemicals for chemoprevention and treatment for cancer, not only in cell lines but also in animal models of cancer (6). Accordingly, we recently showed that phytochemicals such as lycopene and nobiletin possess differential anticancer activity against breast cancer cells with different hormone receptor and HER2 status $(7,8)$.

Pterostilbene (trans-3, 5-dimethoxy-4'-hydroxystilbene) is a stilbenoid phytochemical with similar structure and biological activities to those of resveratrol (9). Like resveratrol, pterostilbene has multiple pharmacological properties, including antioxidant, anti-inflammatory, and cancer-preventive activity (10-12). Although many of the benefits associated with resveratrol also apply to pterostilbene, pterostilbene has a longer half-life and higher oral bioavailability (13). Moreover, it is more potent than resveratrol in inhibiting the growth of human colon cancer cells as well as preventing azoxymethaneinduced colonic tumorigenesis (14). Although the excellent antioxidant property of pterostilbene is most likely the basis for its preventive role against cancer at the initiating and 
progression stages, increasing evidence indicates that pterostilbene has anticancer activity against a variety types of human cancer cells through modulation of diverse molecular targets involving growth factor signaling, cell-cycle regulation, and cell survival/apoptosis (15-17). In animal studies, pterostilbene inhibited the growth of pancreatic and breast cancer in xenotransplanted nude mice $(16,18)$. These in vitro and in vivo studies suggest that pterostilbene might be potentially useful for the prevention and treatment of various types of human cancer. Since phytochemicals have been suggested to lower the risk of ER-negative rather than ERpositive breast cancer, it is of interest to determine whether there are any differences in pterostilbene activity among different subtypes of breast cancer cells. In the present study, we investigated the cellular and molecular mechanisms of the growth-inhibitory activity of pterostilbene against three human breast cancer cell lines that differ in their hormone receptor and HER2 status. In addition, an in vivo experiment using a mouse xenograft model was conducted to determine whether the inhibitory effects of pterostilbene are clinically applicable to human breast cancer.

\section{Materials and Methods}

Cell culture and chemicals. The study was performed on three subtypes of breast cancer cell lines, namely ER-positive MCF-7 cells, hormone receptor-negative but HER2-positive SK-BR-3 cells, and triple-negative MDA-MB-468 cells, all purchased from the American Type Culture Collection (Manassas, VA, USA). Origins of the cell lines and their hormone receptor and HER2 status have been described previously (19). These cell lines were cultured in RPMI-1640 (Wako, Osaka, Japan) supplemented with 10\% fetal bovine serum (Life Technologies, Carlsbad, CA, USA), $100 \mathrm{IU} / \mathrm{ml}$ penicillin and $100 \mu \mathrm{g} / \mathrm{ml}$ streptomycin in a humidified atmosphere of $95 \%$ air and $5 \% \mathrm{CO}_{2}$ at $37^{\circ} \mathrm{C}$. Pterostilbene was purchased from Carbosynth Ltd. (Berkshire, UK), and stored at $-80^{\circ} \mathrm{C}$. A $1.0 \mathrm{M}$ solution was prepared by dissolving original pterostilbene with dimethyl sulfoxide (DMSO; Sigma-Aldrich, St. Louis, MO, USA). A $10 \mathrm{mM}$ solution was prepared by diluting this $1.0 \mathrm{M}$ pterostilbene solution with $99.5 \%$ ethanol (WAKO, Osaka, Japan) immediately before experimental use. The final concentration of DMSO for all experiments and treatments (including vehicle controls, where no drug was added) was maintained at $0.01 \%$ or less. These conditions were found to be non-cytotoxic for at least $72 \mathrm{~h}$.

Determination of growth inhibition. The antiproliferative effects of pterostilbene on these breast cancer cell lines were assessed by Coulter counter (Beckman Coulter, Brea, CA, USA) rather than colorimetric WST-1 cell proliferation assay because we have found disagreement between the actual cell number as measured by Coulter counter and colorimetric assays due to the influence of pterostilbene on optical absorbance. Briefly, $2 \mathrm{ml}$ cell suspension was seeded into $35 \mathrm{~mm}$ dishes at density of $5 \times 10^{4}$ cells per dish and incubated overnight. After addition of $0-100 \mu \mathrm{M}$ pterostilbene, cells were further cultured for $72 \mathrm{~h}$ and the cell number was determined. The relative number of viable cells was determined by comparing the number of treated cells with the corresponding number of vehicle-treated cells taken as $100 \%$. Each experiment was performed using three replicate dishes for each pterostilbene concentration and was carried out independently three times. The $\mathrm{IC}_{50}$ value defined as the concentration needed for a $50 \%$ reduction in cell viability, was determined. The antiproliferative effects of similar concentrations of resveratrol on these breast cancer cell lines were also assessed using the same method as described above.

Cell-cycle analysis and apoptosis measurement. At different times following treatment with $100 \mu \mathrm{M}$ pterostilbene, floating and trypsinized adherent cells were combined, fixed in $70 \%$ ethanol, and stored at $4{ }^{\circ} \mathrm{C}$ prior to cell-cycle analysis. We used this high concentration of pterostilbene, because lower concentration is insufficient to clearly visualize the difference of cell cycle distribution. After removal of ethanol by centrifugation, cells were washed with phosphate-buffered saline and stained with a solution containing RNase A and propidium iodide (Sigma-Aldrich). Cell-cycle analyses were performed on a Beckman Coulter Gallios Flow Cytometer using the Kaluza ver.1.2 software Packages (Beckman Coulter), and the extent of apoptosis was determined by measuring the sub- $\mathrm{G}_{0} / \mathrm{G}_{1}$ population.

Western blot analysis of signaling proteins for cell cycle, growth and apoptosis. Western blot analysis was performed as previously described (20). Equal amounts of proteins prepared by lysed cells treated with $100 \mu \mathrm{M}$ pterostilbene were resolved by $4-15 \%$ sodium dodecylsulfate-polyacrylamide gel electrophoresis (Bio Rad, Hercules, CA, USA) and electrotransferred onto a polyvinylidene difluoride membrane (GE Healthcare, Piscataway, NJ, USA). Nonspecific binding sites were blocked by incubating the membranes in blocking buffer (Nacalai Tesque, Kyoto, Japan) at room temperature for $30 \mathrm{~min}$. The membranes were then incubated with primary antibodies against either phospho-mTOR (Ser2448) (Abcam, Cambridge, UK), phospho-p44/42 ERK1/2 (Thr202/Tyr204), phospho-AKT (Ser473), Cyclin D1 (2992), p21 (C19) (Santa Cruz Biotech, Dallas, TX, USA), BCL-xL (2762), or BAX (2772). The membranes were hybridized with horseradish peroxidase-conjugated secondary antibody. Immunoblots were then developed with the enhanced chemiluminescence system (GE Healthcare) and were then quantified using a LAS-3000 Luminescent Image Analyzer (Fuji Film, Tokyo, Japan). The blots were stripped and reprobed with primary antibodies against mTOR, ERK1/2, AKT and $\beta$-actin. All primary and secondary antibodies, except those for $\mathrm{p} 21$ and phosphomTOR, were purchased from Cell Signaling Technology (Danvers, MA, USA). For reblotting, membranes were incubated in stripping buffer (Thermo, Rockford, IL, USA) for $30 \mathrm{~min}$ at room temperature before washing, blocking, and incubating with antibody. Triplicate determinations were made in separate experiments.

Animal experiments. Animal experiments were carried out in accordance with the protocols approved by the Institutional Animal Care and Use Committee of Nakamura Gakuen University (No. 20156). Two weeks prior to the inoculation of tumor cells, 12 female nude mice (BALB/cAJcl-nu/nu, CLEA Japan) at the age of 5 weeks were divided into two groups each consisting of six mice, and fed modified NIH-07 powder diet devoid of soy isoflavone (NIH-07PLD; Oriental Yeast Co. Ltd, Tokyo, Japan), supplemented with or without $0.1 \%$ pterostilbene $(\mathrm{w} / \mathrm{w})$ throughout the experiments. The composition of modified NIH-07PLD diet was described previously (21). Food intake and bodyweight were monitored throughout the duration of the experiment. Approximately $5 \times 10^{6}$ MDA-MB-468 cells were injected 
subcutaneously into the dorsal flank. The date of appearance and size of palpable tumors were monitored weekly over a period of 8 weeks. Tumors were measured using calipers, and tumor volume was calculated by the formula: tumor volume $\left(\mathrm{mm}^{3}\right)=[$ length $(\mathrm{mm})] \times$ [width $(\mathrm{mm})]^{2} \times 0.52$.

Statistical analysis. To determine the significance of observed differences, analysis of variance (ANOVA) was applied to the data using SPSS statistical software (version 19 for Windows; IBM Corp., Armonk, NY, USA). Time-course analysis data of the cell cycle distribution was compared using Dunnett's test. The mean values of $\mathrm{IC}_{50}$ were compared by Tukey's test. Comparisons of $\mathrm{IC}_{50}$ between pterostilbene and resveratrol were made by Student's $t$-test Data for bodyweight, food intake, and tumor volume in nude mice were analyzed by Student's $t$-test. Differences with a $p$-value less than 0.05 were considered significant.

\section{Results}

Effects of pterostilbene and resveratrol on proliferation and survival. Pterostilbene treatment for 72 consecutive hours led to dose-dependent growth inhibition of MCF-7, SK-BR-3, and MDA-MB-468 cells, with the greatest growth inhibition observed in MDA-MB-468 cells (Figure 1). The $\mathrm{IC}_{50}$ value for MDA-MB-468 cells treated with pterostilbene for $72 \mathrm{~h}$ was significantly lower compared to that for MCF-7 cells, showing approximately 2-fold greater sensitivity to pterostilbene (Table I). HER2-positive SK-BR-3 cells were moderately sensitive to pterostilbene, with an $\mathrm{IC}_{50}$ between those of triple-negative MDA-MB-468 and ER-positive MCF-7 cells. IC $_{50}$ values for resveratrol were similar to those of pterostilbene in the respective breast cancer cell lines, although pterostilbene was significantly more cytotoxic than resveratrol in SK-BR-3 cells (Table I).

Time-course analysis of the effect of pterostilbene on cell-cycle progression and apoptosis. To examine whether the inhibitory effects observed in cytotoxicity assays reflect the arrest or delay of cell-cycle progression or apoptotic cell death, cells were treated with pterostilbene at a dose of $100 \mu \mathrm{M}$, and cellcycle progression and apoptosis were evaluated by fluorescence-activated cell sorting analysis. Treatment with pterostilbene significantly increased the population of $\mathrm{G}_{0} / \mathrm{G}_{1}$ phase cells from $62-64 \%$ at the beginning of the treatment to $70-72 \%$ after $24 \mathrm{~h}$ exposure, with a corresponding decrease in cells in $\mathrm{G}_{2} / \mathrm{M}$ phase, irrespective of the subtype of breast cancer (Figure 2), and the percentages of cells in each cellcycle phase did not significantly change afterwards (Figure 2). Although the percentage of the sub- $\mathrm{G}_{0} / \mathrm{G}_{1}$ cell population, which represents apoptotic cells, increased gradually with time in MCF-7 cells, the sub- $\mathrm{G}_{0} / \mathrm{G}_{1}$ cell population in SK-BR-3 cells significantly increased by approximately three-fold $72 \mathrm{~h}$ post-treatment (Figure 2b). By contrast, the sub- $\mathrm{G}_{0} / \mathrm{G}_{1}$ apoptotic cell population in MDA-MB-468 cells substantially increased after $24 \mathrm{~h}$ exposure (18.1\%) and increased by nearly

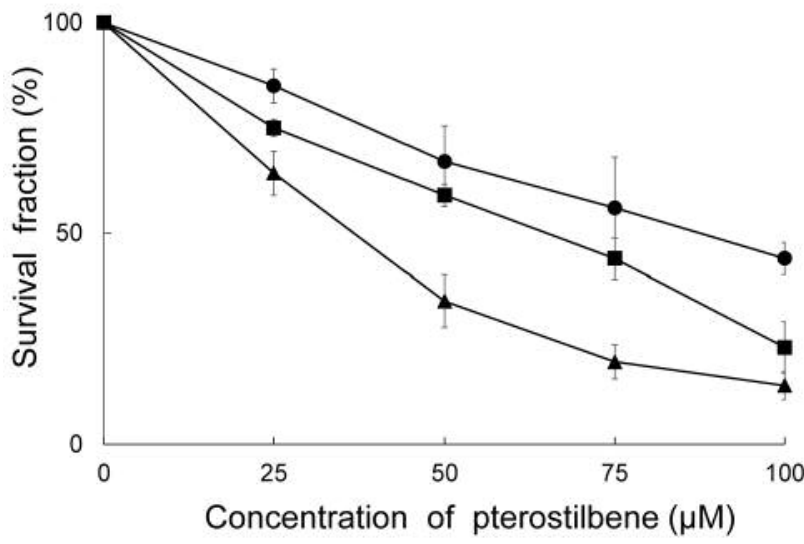

Figure 1. Survival curves for pterostilbene-treated MCF-7 (๑), SK-BR$3(\mathbf{\square})$, and MDA-MB-468 ( $\mathbf{\Delta})$ cells. Cells were treated with different concentrations of pterostilbene for $72 \mathrm{~h}$ and the number of cells was measured by Coulter counter as described in the Materials and Methods. The data represent the means from three independent experiments. Bars=standard deviation $(S D)$.

Table I. The concentrations of pterostilbene and resveratrol needed for a $50 \%$ reduction in cell viability $\left(I C_{50}\right)$ in breast cancer cell lines treated for $72 \mathrm{~h}$. Cells were treated with different concentrations of pterostilbene and resveratrol for $72 \mathrm{~h}$. The $I C_{50}$ values were assessed by Coulter counter as described in the Materials and Methods section. Values are mean $\pm S D$ of three independent experiments. $I C_{50}$ values for pterostilbene and resveratrol for individual cell lines were compared by Tukey's test: $* p<0.05$, $* * p<0.01$. Comparisons of $I C_{50}$ between pterostilbene and resveratrol were made by Student's t-test.

\begin{tabular}{|c|c|c|c|c|}
\hline \multirow{2}{*}{ Cell line } & \multicolumn{3}{|c|}{$\mathrm{IC}_{50}(\mu \mathrm{M})$} & \multirow{2}{*}{$\begin{array}{c}p \text {-Value } \\
\text { pterostilbene } \\
\text { versus resveratrol }\end{array}$} \\
\hline & Pterostilbe & Resveratro & & \\
\hline MCF-7 & $87.6 \pm 9.0$ & $88.8 \pm 6.7$ & & 0.863 \\
\hline SK-BR-3 & $64.4 \pm 4.6$ & $75.3 \pm 3.1$ & & 0.026 \\
\hline MDA-MB-468 & $45.7 \pm 5.2$ & $49.3 \pm 1.2$ & & 0.309 \\
\hline
\end{tabular}

two-fold after $72 \mathrm{~h}$ exposure, resulting in $35.8 \%$ at which the greatest growth inhibition was observed (Figure 2c). These data indicate that pterostilbene-induced growth decline in SKBR-3 and MDA-MB-468 cells appears to be mediated mainly by progressive expansion of the apoptotic cell population.

Effect of pterostilbene on activation of signaling molecules. Because activation of signaling molecules such as ERK1/2, AKT and mTOR have been considered as major factors contributing to proliferation and survival, we examined the effect of pterostilbene on expression or activation 

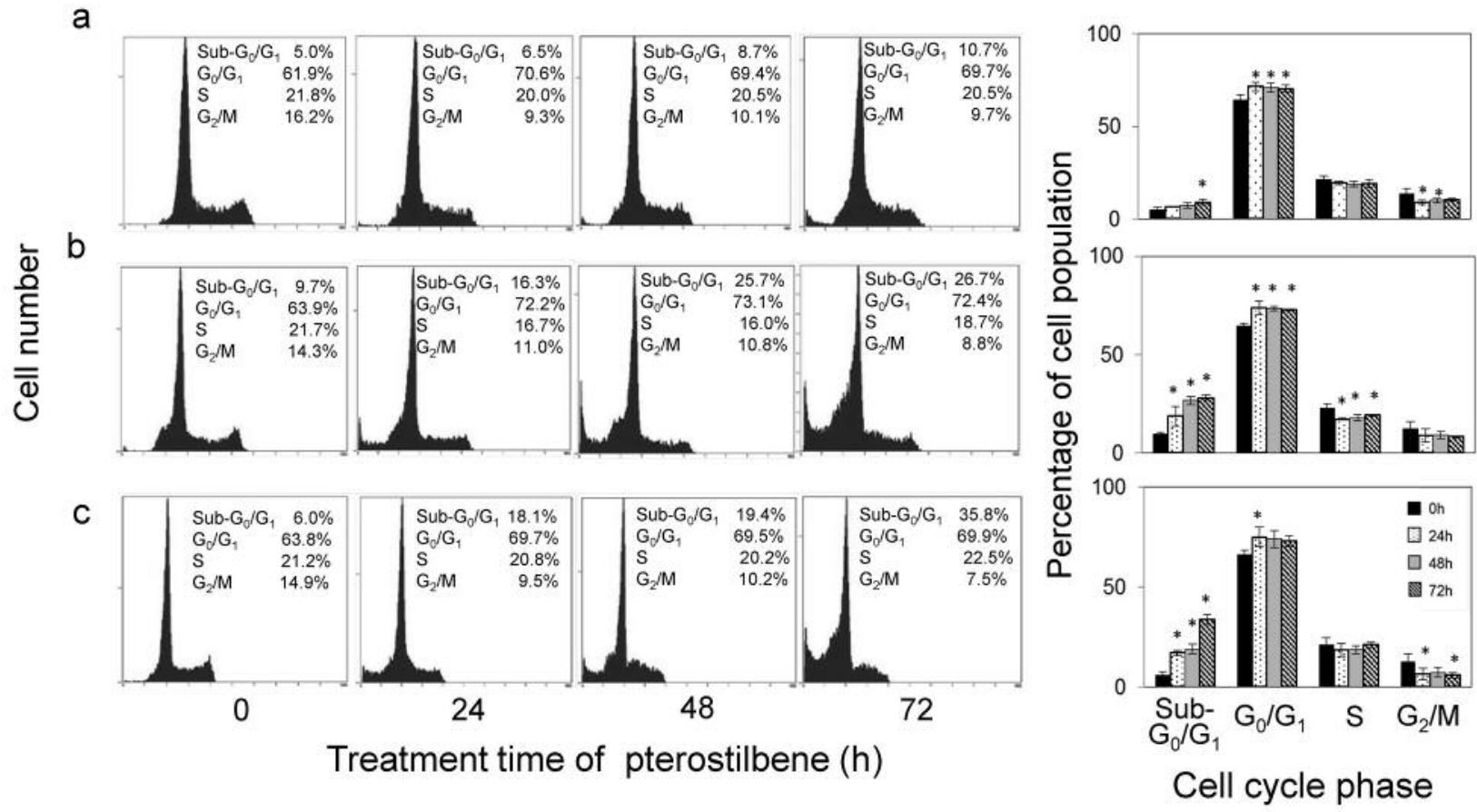

Figure 2. Time-course analysis of the effect of pterostilbene on cell-cycle progression and apoptosis as determined by flow cytometry. Representative cell-cycle distributions after exposure of MCF-7 (a), SK-BR-3 (b) and MDA-MB-468 (c) breast cancer cell lines to 100 $\mu M$ pterostilbene for 0, 24, 48 and $72 \mathrm{~h}$. Percentages of the total cell population in the different phase of cell cycle are also shown in the right panels of each cell line. Data are the means $\pm S D$ of three independent experiments. *Significant difference versus control $(0 h)$ at $p<0.05$.

(phosphorylation) of these proteins. Constitutive activity of ERK1/2 was enhanced $6 \mathrm{~h}$ after initiation of treatment with pterostilbene in SK-BR-3 and MDA-MB-468 cells, and remained at high levels over $24 \mathrm{~h}$ (Figure $3 \mathrm{a}$ ). In contrast, the phosphorylation of AKT and its downstream molecule mTOR was inhibited time-dependently in MDA-MB-468 cells, and slightly inhibited in SK-BR-3 cells but not in MCF-7 cells (Figure 3a). Cyclin D1, functioning as a key regulator of the $G_{0} / G_{1}$ cell-cycle checkpoint, was inhibited with subsequent increase of $\mathrm{p} 21$ protein in all three subtypes of breast cancer cells, indicating that pterostilbene arrests the cell cycle at $G_{0} / G_{1}$ through a decrease in cyclin D1 expression and concomitant up-regulation of p21 regardless of breast cancer subtype.

Effects of pterostilbene on expression of pro- and anti-apoptotic proteins. To elucidate the apoptotic mechanism induced by pterostilbene, we examined the expression of anti-apoptotic BCL-xL as well as pro-apoptotic BAX (Figure 3b). Upon treatment with $100 \mu \mathrm{M}$ pterostilbene, BCL-xL expression did not change in any of the subtypes of breast cancer. By contrast, expression of BAX slightly increased with incubation time with pterostilbene in MCF-7, but was substantially enhanced in SK-BR-3 and MDA-MB-468 cells. Therefore, enhanced expression of BAX appears to be responsible for pterostilbeneinduced apoptotic induction in those breast cancer cell lines. A slight increase in BAX expression in MCF-7 cells might explain the lower apoptosis induction in these cells compared to SK-BR-3 and MDA-MB-468 cells.

Effects of pterostilbene on MDA-MB-468 cells xenotransplanted into nude mice. MDA-MB-468 triple-negative cells were found to be most sensitive to pterostilbene. Therefore, we conducted an in vivo experiment using nude mice xenotransplanted with MDA-MB-468 cells to determine whether the inhibitory effects of pterostilbene were clinically applicable to human breast cancer. There were no significant differences of weight gain and consumption of food between mice fed the control diet and those fed pterostilbene-containing diet over 10 weeks (Table II), suggesting no signs of toxicity induced by pterostilbene. Although tumors grew rapidly in mice fed the control diet, addition of pterostilbene apparently suppressed tumor growth over 10 weeks (Figure 4), reducing the tumor size by $76 \%$ compared to the control diet (Table II). These data indicate that pterostilbene substantially inhibits tumor growth in vivo without apparent toxicity. 


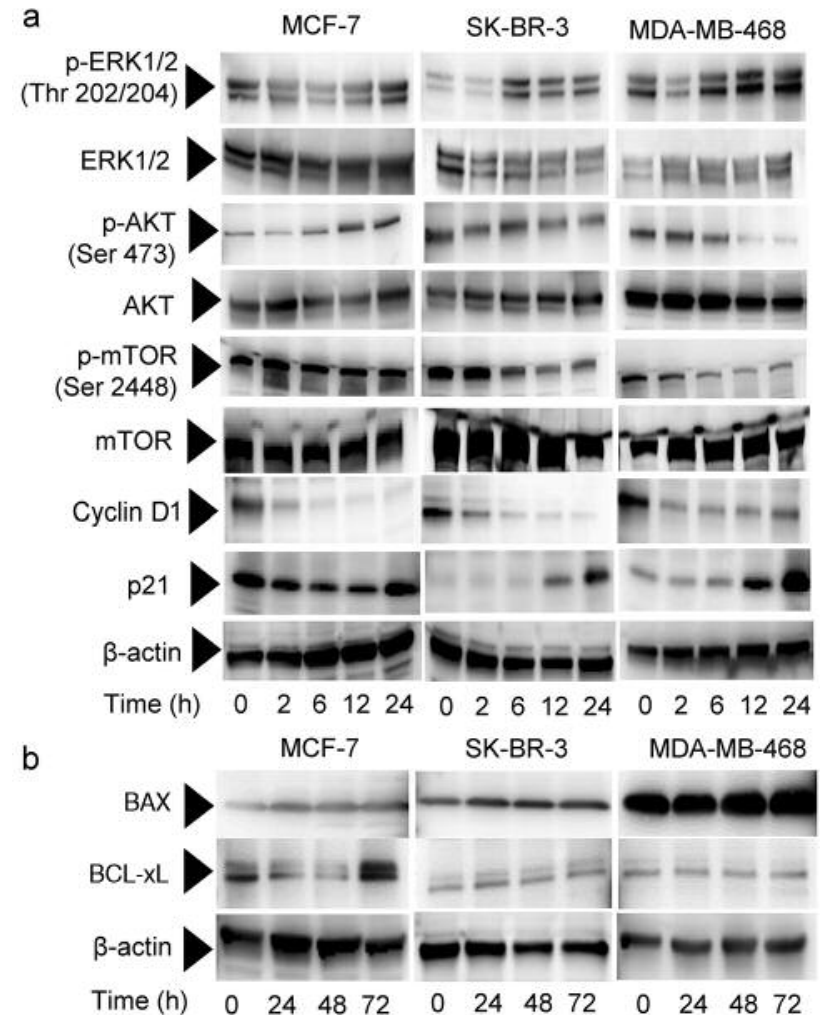

Figure 3. Effects of pterostilbene on activation of signaling molecules for cell proliferation and survival. Cells were treated with $100 \mu \mathrm{M}$ pterostilbene for the indicated times and harvested for western blot analysis. (a) Western blots are shown for phosphorylated and total ERK1/2, AKT, and mTOR. Cyclin D1 and p21 are also shown. (b) Effects of pterostilbene on apoptotic and anti-apoptotic proteins $B A X$ and BCL-xL. $\beta$-Actin was used as an internal control.

\section{Discussion}

In the present study, we found that pterostilbene has differential anticancer activity against three subtypes of human breast cancer that differ in their hormone receptor and HER2 status. Pterostilbene exhibited a dose-dependent growth-inhibitory activity, with the greatest inhibition observed in triple-negative MDA-MB-468 cells. ER-positive MCF-7 cells were the least sensitive to pterostilbene, while HER2-positive SK-BR-3 cells were moderately sensitive. Compared to resveratrol, the $\mathrm{IC}_{50}$ values for pterostilbene were similar to those of resveratrol in the three respective subtypes of breast cancer cells, indicating that pterostilbene and resveratrol exhibit almost equivalent anticancer activity. In contrast to our data, a previous report showed pterostilbene to be more potent than resveratrol in inhibiting the growth of human colon cancer cells (14). Therefore, the anticancer activity of these resveratrol derivatives may vary depending on the cancer cell origin. Because of its longer a

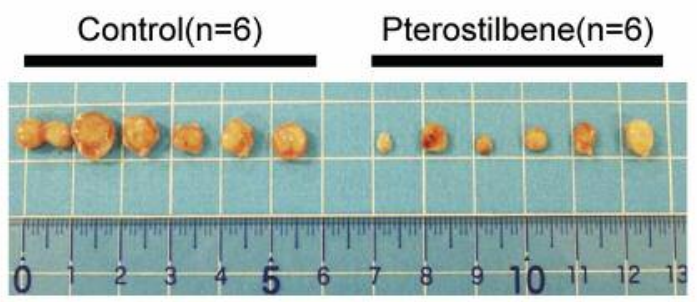

b

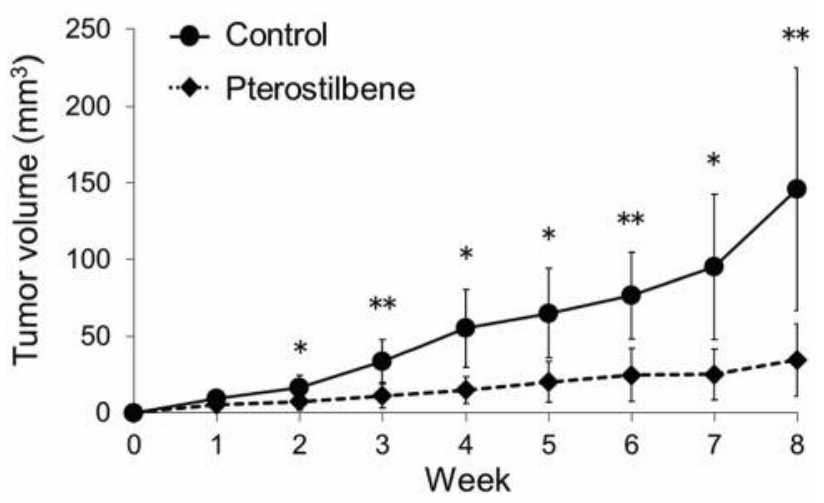

Figure 4. Effects of pterostilbene on tumor from MDA-MB-468 cells xenotransplanted into nude mice. The experimental treatment protocol is described in the Materials and Methods section. a: Tumors excised from six individual mice in each group are shown. b: Time course changes of the mean tumor volume in control mice and pterostilbenetreated mice implanted with MDA-MB-468 cells. Six samples were analyzed in each group, and values represent the mean $\pm S D$. Significantly different at $* p<0.05$, and $* * p<0.01$.

Table II. Bodyweight, tumor volume, and total food consumption of mice xenotransplanted with MDA-MB-468 cells.

\begin{tabular}{lccc}
\hline & $\begin{array}{c}\text { Control } \\
(\mathrm{n}=6)\end{array}$ & $\begin{array}{c}\text { Pterostilbene } \\
(\mathrm{n}=6)\end{array}$ & $p$-Value \\
\hline Bodyweight $(\mathrm{g})^{\mathrm{b}}$ & $25.1 \pm 1.3$ & $25.3 \pm 1.9$ & 0.625 \\
${\text { Tumor volume }\left(\mathrm{mm}^{3}\right)^{\mathrm{b}}}$ & $146.0 \pm 79.3$ & $34.6 \pm 23.7$ & 0.008 \\
Food intake $(\mathrm{g})^{\mathrm{c}}$ & $311.0 \pm 15.2$ & $327.8 \pm 19.3$ & 0.305 \\
\hline
\end{tabular}

Data are presented as the mean $\pm \mathrm{SD}$. aStudent's $t$-test, control versus

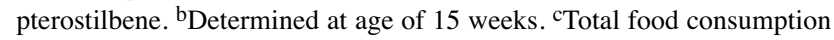
during experiment.

half-life and higher oral bioavailability than resveratrol (13), pterostilbene would be superior as a potential preventive phytochemical for breast cancer overall and, more specifically, ER-negative breast cancer such as HER2positive and triple-negative breast cancer. Moreover, using 
nude mice xenotransplanted with triple-negative MDA-MB468 cells, pterostilbene substantially inhibited tumor growth without apparent toxicity, suggesting a potential clinical application in humans. Correspondingly, pterostilbene has been shown to be generally safe for use in humans up to $250 \mathrm{mg}$ /day (22). Taken together, these data suggest the therapeutic usefulness of pterostilbene in the treatment of human breast cancer, thus warranting clinical evaluation of pterostilbene in patients with triple-negative breast cancer.

In the present study, pterostilbene induced a substantial delay of the cell cycle at $G_{0} / G_{1}$ phase regardless of breast cancer subtype, and reduced the expression of cyclin D1, with a concomitant increase of $\mathrm{p} 21$ protein. Previous studies reported that pterostilbene induced $\mathrm{G}_{0} / \mathrm{G}_{1}$ cell-cycle arrest, corroborated by the down-regulation of cyclin D1 with/without up-regulation of p21 in several human cancer cell lines including human prostate (23), gastric (17), and hormone-dependent mammary cancer (24) cells. Our results, together with the previous findings, suggest that the arrest of the cell cycle at $G_{0} / G_{1}$ through down-regulation of cyclin D1 and/or up-regulation of p21 might be a universal event triggered by pterostilbene treatment. The molecular mechanism whereby pterostilbene arrests cells in the $G_{0} / G_{1}$ phase remains unclear. However, enhancement of constitutive activity of ERK1/2 may cause pterostilbene-induced $G_{0} / G_{1}$ block as demonstrated in breast cancer cells treated with lycopene (8). ERK1/2 is an important subfamily of mitogenactivated protein kinases that controls a broad range of cellular activities and physiological processes (25). Activation of ERK1/2 generally has proliferative capacity, but under certain conditions, ERK $1 / 2$ has been shown to have antiproliferative activity through $\mathrm{G}_{1}$ cell-cycle arrest (26). A robust early phase of ERK1/2 signaling followed by a moderate sustained phase leads to transient induction of p21 and accumulation of cyclin D1, allowing $\mathrm{G}_{1}$ progression. However, robust and prolonged activation of ERK1/2 causes $\mathrm{G}_{1}$ arrest due to long-term p21 induction and cyclindependent kinase 2 inhibition (26). In the present study, continuous treatment with pterostilbene enhanced the constitutive activity of ERK1/2 from $6 \mathrm{~h}$, which remained at high levels with p21 induction over $24 \mathrm{~h}$ in SK-BR-3 and MDA-MB-468 cells. Therefore, pterostilbene-induced strong and sustained activation of ERK pathway may cause $G_{0} / G_{1}$ cell cycle blockade in breast cancer cells.

We found that the growth-inhibitory activity of pterostilbene is mediated by differential mechanisms of action, depending on ER and HER2 status. Pterostilbene substantially induced apoptotic cell death by increasing the expression of BAX, without affecting the BCL-xL level in SK-BR-3 and MDA-MB-468 cells. In contrast, ER-positive MCF-7 cells, which were found to be twofold more resistant to pterostilbene than triple-negative MDA-MB-468 cells, showed marginal induction of apoptosis, with slight upregulation of BAX. It has been suggested that AKT plays a critical role in controlling survival and apoptosis through direct phosphorylation of mTOR, thereby inducing signals leading to anti-apoptotic pathway. In this study, pterostilbene clearly inactivated AKT and downstream mTOR in triplenegative MDA-MB-468 cells, but not in ER-positive cells. Therefore, these data suggest that pterostilbene may execute its apoptotic effect primarily through blocking AKT/mTORmediated signaling pathways in triple-negative cells. Similar pterostilbene-induced apoptosis through suppression of AKT signaling pathway has been reported in breast cancer and colon cancer cell lines $(16,27)$. Furthermore, pterostilbene also induces apoptosis in human oral cancer cell lines by reducing phosphorylation of AKT and mTOR (28). Taken together, pterostilbene induces apoptosis by enhancing the expression of BAX through inactivation of AKT and mTOR, indicating the important role of BAX in pterostilbene-induced apoptosis induction. Although additional research is needed to identify the mechanisms accounting for the lower sensitivity of ER-positive cells to pterostilbene, our data may support the epidemiological evidence that fruit and vegetable intake reduces the risk of ER-negative rather than ER-positive breast cancer $(4,5)$.

Despite recent advances in breast cancer treatment, breast cancer recurrence is a major problem and represents the principal cause of breast cancer-related death. Emerging evidence suggests the existence of cancer stem cells, a population of cells capable of self-renew and initiating tumor growth, which might be responsible for breast cancer recurrence (29). Recently, phytochemicals have gained immense attention because of their wide safety profile and ability to target heterogeneous populations of cancer cells, including cancer stem cells (30). Moreover, pterostilbene has been reported to selectively kill breast cancer stem cells isolated form MCF-7 cells (31). In our study, pterostilbene inhibited the growth of xenotransplanted triple-negative MDA-MB-468 tumors consisting of a population of in vivoselected and thus highly tumorigenic cells resembling cancer stem cells. In this regard, our data suggest that pterostilbene might be potentially useful not only for prevention and treatment, but also the reduction of recurrence of human breast cancer, especially triple-negative breast cancer.

\section{Conflicts of Interest}

The Authors have no conflict of interest in regard to this study.

\section{Acknowledgements}

This work was supported by JSPS KAKENHI Grant Numbers 15K00864 and 26750059, and the Science Research Promotion Fund from the Promotion and Mutual Aid Corporation for Private Schools of Japan. This work was also supported by the Cancer Research Fund from Fukuoka Foundation for Sound Health. 


\section{References}

1 Torre LA, Bray F, Siegel RL, Ferlay J, Lortet-Tieulent J and Jemal A: Global cancer statistics, 2012. CA Cancer J Clin 65(2): 87-108, 2015.

2 Cetin I and Topcul M: Triple-negative breast cancer. Asian Pac J Cancer Prev 15(6): 2427-2431, 2014.

3 Aune D, Chan DS, Vieira AR, Rosenblatt DA, Vieira R, Greenwood DC and Norat T: Fruits, vegetables and breast cancer risk: A systematic review and meta-analysis of prospective studies. Breast Cancer Res Treat 134(2): 479-493, 2012.

4 Fung TT, Hu FB, McCullough ML, Newby PK, Willett WC and Holmes MD: Diet quality is associated with the risk of estrogen receptor-negative breast cancer in postmenopausal women. J Nutr 136(2): 466-472, 2006.

5 Farvid MS, Chen WY, Michels KB, Cho E, Willett WC and Eliassen AH: Fruit and vegetable consumption in adolescence and early adulthood and risk of breast cancer: Population based cohort study. BMJ 353: i2343, 2016.

6 Bathaie SZ, Faridi N, Nasimian A, Heidarzadeh H and Tamanoi F: How phytochemicals prevent chemical carcinogens and/or suppress tumor growth. Enzymes 37: 1-42, 2015.

7 Chen C, Ono M, Takeshima M and Nakano S: Antiproliferative and apoptosis-inducing activity of nobiletin against three subtypes of human breast cancer cell lines. Anticancer Res 34(4): 1785-1792, 2014.

8 Takeshima M, Ono M, Higuchi T, Chen C, Hara T and Nakano $\mathrm{S}$ : Anti-proliferative and apoptosis-inducing activity of lycopene against three subtypes of human breast cancer cell lines. Cancer Sci 105(3): 252-257, 2014.

9 Estrela JM, Ortega A, Mena S, Rodriguez ML and Asensi M: Pterostilbene: Biomedical applications. Crit Rev Clin Lab Sci 50(3): 65-78, 2013.

10 McCormack D and McFadden D: A review of pterostilbene antioxidant activity and disease modification. Oxid Med Cell Longev 2013: 575482, 2013.

11 Paul S, Rimando AM, Lee HJ, Ji Y, Reddy BS and Suh N: Antiinflammatory action of pterostilbene is mediated through the p38 mitogen-activated protein kinase pathway in colon cancer cells Cancer Prev Res 2(7): 650-657, 2009.

12 Rimando AM, Cuendet M, Desmarchelier C, Mehta RG, Pezzuto JM and Duke SO: Cancer chemopreventive and antioxidant activities of pterostilbene, a naturally occurring analogue of resveratrol. J Agric Food Chem 50(12): 3453-3457, 2002.

13 Kapetanovic IM, Muzzio M, Huang Z, Thompson TN and McCormick DL: Pharmacokinetics, oral bioavailability, and metabolic profile of resveratrol and its dimethylether analog, pterostilbene, in rats. Cancer Chemother Pharmacol 68(3): 593 601, 2011.

14 Chiou YS, Tsai ML, Nagabhushanam K, Wang YJ, Wu CH, Ho $\mathrm{CT}$ and Pan MH: Pterostilbene is more potent than resveratrol in preventing azoxymethane (AOM)-induced colon tumorigenesis via activation of the NF-E2-related factor 2 (NRF2)-mediated antioxidant signaling pathway. J Agric Food Chem 59(6): 27252733, 2011.

15 Mannal PW, Alosi JA, Schneider JG, McDonald DE and McFadden DW: Pterostilbene inhibits pancreatic cancer in vitro. J Gastrointest Surg 14(5): 873-879, 2010.

16 Pan C, Hu Y, Li J, Wang Z, Huang J, Zhang S and Ding L: Estrogen receptor-alpha36 is involved in pterostilbene-induced apoptosis and anti-proliferation in in vitro and in vivo breast cancer. PLoS One 9(8): e104459, 2014

17 Pan MH, Chang YH, Badmaev V, Nagabhushanam K and Ho CT: Pterostilbene induces apoptosis and cell-cycle arrest in human gastric carcinoma cells. J Agric Food Chem 55(19): 7777-7785, 2007.

18 McCormack DE, Mannal P, McDonald D, Tighe S, Hanson J and McFadden D: Genomic analysis of pterostilbene predicts its antiproliferative effects against pancreatic cancer in vitro and in vivo. J Gastrointest Surg 16(6): 1136-1143, 2012.

19 Neve RM, Chin K, Fridlyand J, Yeh J, Baehner FL, Fevr T, Clark L, Bayani N, Coppe JP, Tong F, Speed T, Spellman PT, DeVries S, Lapuk A, Wang NJ, Kuo WL, Stilwell JL, Pinkel D, Albertson DG, Waldman FM, McCormick F, Dickson RB, Johnson MD, Lippman M, Ethier S, Gazdar A and Gray JW: A collection of breast cancer cell lines for the study of functionally distinct cancer subtypes. Cancer Cell 10(6): 515-527, 2006.

20 Ono M, Higuchi T, Takeshima M, Chen $\mathrm{C}$ and Nakano S: Differential antitumor activities of curcumin against RAS- and SRC-activated human adenocarcinoma cells. Biochem Biophys Res Commun 436(2): 186-191, 2013.

21 Ono M, Koga T, Ueo H and Nakano S: Effects of dietary genistein on hormone-dependent rat mammary carcinogenesis induced by ethyl methanesulphonate. Nutr Cancer 64(8): 1204-1210, 2012.

22 Riche DM, McEwen CL, Riche KD, Sherman JJ, Wofford MR, Deschamp D and Griswold M: Analysis of safety from a human clinical trial with pterostilbene. J Toxicol 2013: 463595, 2013.

23 Lin VC, Tsai YC, Lin JN, Fan LL, Pan MH, Ho CT, Wu JY and Way TD: Activation of ampk by pterostilbene suppresses lipogenesis and cell-cycle progression in p53-positive and negative human prostate cancer cells. J Agric Food Chem 60(25): 6399-6407, 2012.

24 Wang Y, Ding L, Wang X, Zhang J, Han W, Feng L, Sun J, Jin $\mathrm{H}$ and Wang $\mathrm{XJ}$ : Pterostilbene simultaneously induces apoptosis, cell-cycle arrest and cytoprotective autophagy in breast cancer cells. Am J Transl Res 4(1): 44-51, 2012.

25 Brown MD and Sacks DB: Protein scaffolds in map kinase signalling. Cell Signal 21(4): 462-469, 2009.

26 Meloche S and Pouyssegur J: The ERK1/2 mitogen-activated protein kinase pathway as a master regulator of the G1- to Sphase transition. Oncogene 26(22): 3227-3239, 2007.

27 Tolba MF and Abdel-Rahman SZ: Pterostilbine, an active component of blueberries, sensitizes colon cancer cells to 5fluorouracil cytotoxicity. Sci Rep 5: 15239, 2015.

28 Ko CP, Lin CW, Chen MK, Yang SF, Chiou HL and Hsieh MJ: Pterostilbene induce autophagy on human oral cancer cells through modulation of AKT and mitogen-activated protein kinase pathway. Oral Oncol 51(6): 593-601, 2015.

29 Ahmad A: Pathways to breast cancer recurrence. ISRN Oncol 2013: 290568, 2013

30 Dandawate PR, Subramaniam D, Jensen RA and Anant S: Targeting cancer stem cells and signaling pathways by phytochemicals: Novel approach for breast cancer therapy. Semin Cancer Biol 40-41: 192-208, 2016.

$31 \mathrm{Wu} \mathrm{CH}$, Hong BH, Ho CT and Yen GC: Targeting cancer stem cells in breast cancer: Potential anticancer properties of 6-shogaol and pterostilbene. J Agric Food Chem 63(9): 2432-2441, 2015.

Received July 30, 2017

Revised August 13, 2017

Accepted August 22, 2017 